\title{
PERSPEX MACHINE VIII: \\ AXIOMS OF TRANSREAL ARITHMETIC
}

\begin{abstract}
Notice
This is the published version of the paper that will appear in SPIE as noted below. It replaces an earlier, pre-publication, version of the paper.
\end{abstract}

\section{COPYRIGHT}

Copyright 2006 Society of Photo-Optical Instrumentation Engineers. This paper will be published in Vision Geometry XV, Longin Jan Lateki, David M. Mount, Angela Y. Wu, Editors, Proceedings of SPIE Vol. 6499 (2007) and is made available as an electronic copy with permission of SPIE. One print or electronic copy may be made for personal use only. Systematic or multiple reproduction, distribution to multiple locations via electronic or other means, duplication of any material in this paper for a fee or for commercial purposes, or modifications of the content of the paper are prohibited. 


\title{
Perspex Machine VIII: Axioms of Transreal Arithmetic
}

\author{
James A.D.W. Anderson, ${ }^{* a}$ Norbert Völker, ${ }^{\mathrm{b}}$ Andrew A. Adams, ${ }^{\mathrm{a}}$ \\ ${ }^{a}$ Computer Science, University of Reading, England \\ ${ }^{\mathrm{b}}$ Computer Science, University of Essex, England
}

\begin{abstract}
Transreal arithmetic is a total arithmetic that contains real arithmetic, but which has no arithmetical exceptions. It allows the specification of the Universal Perspex Machine which unifies geometry with the Turing Machine. Here we axiomatise the algebraic structure of transreal arithmetic so that it provides a total arithmetic on any appropriate set of numbers. This opens up the possibility of specifying a version of floating-point arithmetic that does not have any arithmetical exceptions and in which every number is a first-class citizen.
\end{abstract}

We find that literal numbers in the axioms are distinct. In other words, the axiomatisation does not require special axioms to force non-triviality. It follows that transreal arithmetic must be defined on a set of numbers that contains $\{-\infty,-1,0,1, \infty, \Phi\}$ as a proper subset. We note that the axioms have been shown to be consistent by machine proof.

Keywords: transrational arithmetic, transreal arithmetic.

\section{Introduction}

Transreal arithmetic is a generalisation of transrational arithmetic. ${ }^{2}$ It is a total arithmetic that contains real arithmetic, but which has no arithmetical exceptions. ${ }^{2}$ It allows the specification of the Universal Perspex Machine ${ }^{2}$ which unifies geometry with the Turing Machine. Here we axiomatise the algebraic structure of transreal arithmetic so that it provides a total arithmetic on any appropriate set. We expect that the set of transinteger numbers, $Z^{*}=Z \cup\{-\infty, \infty, \Phi\}$, transrational numbers, $Q^{*}=Q \cup\{-\infty, \infty, \Phi\}$, and the set of transreal numbers, $R^{*}=R \cup\{-\infty, \infty, \Phi\}$, will be of most immediate use. Having obtained the transreal numbers the complex, quaternion, and octonion numbers can be extended to total systems of arithmetic by replacing the components of these numbers with transreal numbers. Here $\pm \infty$ are distinct infinities at the extremes of the real number line and nullity, $\Phi$, is a number which lies off the real number line (and off the complex, quaternion, and octonion (hyper)planes). Having $\Phi$ lie off the number line blocks the counter-proofs from real analysis that attempt to show that $0 / 0$ is undefined. In transreal arithmetic division is defined via the reciprocal, not via the multiplicative inverse. The reciprocal contains the multiplicative inverse as a proper subset, but also defines the reciprocal of zero. Hence, transreal arithmetic has an algebraic structure that contains a field as a proper subset, but which does define division by zero. Thus, the usual objections to allowing division by zero are removed. The intention is that the added complexity of the number system is justified by its theoretical and practical advantages for machine computation.

The axiomatisation defines all of the arithmetical operations:,,,$+-+ \quad \div$. It also defines the ordering relationships: $<,=,>$. The definitions are made in terms of an arbitrary element $a$ of some set of numbers $A$ which includes the special numbers $-\infty,-1,0,1, \infty, \Phi$. The axiomatisation has been checked by machine using Isabelle/ HOL. ${ }^{10,11}$ Some hand proofs are given to illustrate the algebraic structure of the transreals.

After the axioms have been presented a number of definitions and theorems are given to facilitate arithmetical work. During the derivation of these theorems we find that literal numbers in the axioms are distinct. In other words, the axiomatisation does not require special axioms to force non-triviality. It follows that transreal arithmetic is defined on a set that contains $\{-\infty,-1,0,1, \infty, \Phi\}$ as a proper subset.

* Corresponding author. author@bookofparagon.com, http://www.bookofparagon.com Computer Science, The University of Reading, Reading, Berkshire, England, RG6 6AY. 


\section{Axiomatisation}

The following meta-symbols are used in the axiomatisation: comma $($,$) ; when (:)$; not equal $(\neq)$; not $(\neg)$; and $(\wedge)$; or $(\vee)$; plus or minus $( \pm)$; single implication $(\Rightarrow)$; bi-implication $(\Leftrightarrow)$. In this axiomatisation the sets of positive and negative numbers are given by the ordering axioms.

The axiomatisation also uses the function $s g n$ as defined next. This definition makes no assumption about distinctness of transreal numbers, instead it relies on evaluating the conditions in reading (program) order. The definition used here is different from the programmatic definition of $\operatorname{sgn}$ given in. ${ }^{4}$ It is a shorthand that is used to state axiom [A31] concisely. It is not essential to the axiomatisation. Nonetheless, the properties of $s g n$ are checked by machine in theorems [T44] to [T47] inclusive.

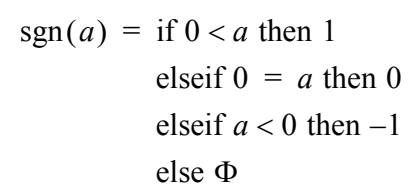

The axiomatisation uses thirty two axioms. Twenty two axioms are analogues of the standard axioms of arithmetic, including the arithmetic of cardinal numbers, and geometrical models of the real-number line. These are: [A1], [A2], [A3], [A5], [A6], [A7], [A8], [A10], [A12], [A13], [A14], [A17], [A18], [A19], [A25], [A26], [A27], [A28], [A29], [A30], [A31], [A32]. Ten axioms always involve strictly transreal numbers. These are: [A4], [A9], [A11], [A15], [A16], [A20], [A21], [A22], [A23], [A24].

\subsection{Axioms}

Additive Associativity

Additive Commutativity

Additive Identity

Additive Nullity

Additive Infinity

Subtraction as Sum with Opposite

Bijectivity of Opposite

Additive Inverse

Opposite of Nullity

Non-null Subtraction of Infinity

Subtraction of Infinity from Infinity

Multiplicative Associativity

Multiplicative Commutativity

Multiplicative Identity

$$
\begin{gathered}
a+(b+c)=(a+b)+c \\
a+b=b+a \\
0+a=a \\
\Phi+a=\Phi
\end{gathered}
$$$$
a+\infty=\infty: a \neq-\infty, \Phi
$$$$
a-b=a+(-b)
$$$$
-(-a)=a
$$$$
a-a=0: a \neq \pm \infty, \Phi
$$$$
-\Phi=\Phi
$$ 
Multiplicative Nullity

Infinity Times Zero

Division

Multiplicative Inverse

Bijectivity of Reciprocal

Reciprocal of Zero

Reciprocal of the Opposite of Infinity

Reciprocal of Nullity

Positive

Negative

Positive Infinity

Ordering

Less Than

Greater Than or Equal

Less Than or Equal

Quadrachotomy

$$
\text { Exactly one of: }(a<0),(a=0),(a>0),(a=\Phi)
$$

Distributivity

$$
a \times(b+c)=(a \times b)+(a \times c): \neg((a= \pm \infty) \wedge(\operatorname{sgn}(b) \neq \operatorname{sgn}(c)) \wedge(b+c \neq 0, \Phi))
$$

Lattice Completeness The set, $X$, of all transreal numbers, excluding $\Phi$, is lattice complete because

$$
\forall Y: Y \subseteq X \Rightarrow(\exists u \in X:(\forall y \in Y: y \leq u) \wedge(\forall v \in X:(\forall y \in Y: y \leq v) \Rightarrow u \leq v))
$$

[A15]

\subsection{Definitions}

The transreals, $R^{\mathrm{T}}$, are all those numbers that satisfy axioms [A1] to [A32]. We now define subsets of the transreals corresponding to the usual number systems $Z, Q, R$. The standard integers, $Z$, are generated from the set $\{0,1\}$ by addition and subtraction. The set of standard rationals, $Q$, is the set of all fractions $n / d$ where $n, d \in Z$ and $d \neq 0$. The set of standard reals, $R$, is the set of all transreals excluding the strictly transreal numbers $\{-\infty, \infty, \Phi\}$.

Reals

$$
R=R^{\mathrm{T}} \backslash\{-\infty, \infty, \Phi\}
$$

Transintegers

$$
Z^{\mathrm{T}}=Z \cup\{-\infty, \infty, \Phi\}
$$

Transrationals

$$
Q^{\mathrm{T}}=Q \cup\{-\infty, \infty, \Phi\}
$$


The transintegers, $Z^{\mathrm{T}}$, are closed over addition, subtraction, and multiplication. These operations are subject to the axioms: [A1] to [A16], [A23] to [A31].

The transrationals, $Q^{\mathrm{T}}$, are closed over addition, subtraction, multiplication, and reciprocal. These operations are subject to the axioms: [A1] to [A31].

The extended reals, $R^{*}=R \cup\{-\infty, \infty\}$, form a complete lattice by axiom [A32]. This implies that $R$ is a complete ordered field and hence is isomorphic to the standard reals.

\subsection{Consistency}

Consistency of the transreal axioms was shown by constructing a model. The construction of the model begins with the set $R$ and the standard arithmetic operations of addition, subtraction, multiplication, and division. The strictly transreal elements are added to the set giving $R^{\mathrm{T}}=R \cup\{-\infty, \infty, \Phi\}$. The arithmetic operations are extended to $R^{\mathrm{T}}$ by suitable definitions. Finally, division by zero is defined. The validity of each of the transreal axioms is then established for this model. The model construction and validity checking were performed in the mechanical proof system Isabelle/HOL. ${ }^{10,11}$ The proof files are available on the world wide web. ${ }^{12}$

\section{Theorems}

In this section we list the theorems that were obtained during the mechanical verification of the properties of transreal arithmetic. Further details of these proofs are given in the proof files. ${ }^{12}$ We then give some hand derivations to illustrate algebraic structure and proof methods in a form that is accessible to the general reader. At the time of writing, very few of the hand proofs have been checked mechanically.

\subsection{Machine Derivations}

The aim of the machine derivations was to establish various elementary, arithmetical, properties of the transreals, including the distinctness of $-\infty,-1,0,1, \infty, \Phi$ and the closure of the positive transreals over addition, multiplication, and reciprocal.

\subsubsection{Simple algebraic properties}

$$
\begin{aligned}
& a+0=a \\
& a+\Phi=\Phi \\
& a-(-b)=a+b \\
& a-\Phi=\Phi \\
& 0-a=-a \\
& \infty+a=\infty: a \neq-\infty, \Phi \\
& a \times 1=a \\
& 0 \times \infty=\Phi \\
& (-a=-b) \equiv(a=b) \\
& (-a+b) \equiv(b-a) \\
& a+b-b=a: b \neq \pm \infty, \Phi
\end{aligned}
$$


$a-b+b=a: b \neq \pm \infty, \Phi$

$-a+a=0: a \neq \pm \infty, \Phi$

$(-a=\Phi) \equiv(a=\Phi)$

$(-a=\infty) \equiv(a=-\infty)$

$\infty-a=\infty:(a \neq \infty) \wedge(a \neq \Phi)$

[T16]

$-a-b=-b-a$

3.1.2 Ordering and distinctness on $-\infty,-1,0,1, \infty, \Phi$

$\neg(0<0)$

$0 \neq \Phi$

$0 \neq \infty$

$\neg(\Phi<0)$

$\neg(0<\Phi)$

$\infty \neq \Phi$

$0<1$

$\neg(1<0)$

$0 \neq 1$

$1 \neq \infty$

$1 \neq \Phi$

$-1<0$

$-1 \times \infty=-\infty$

$0 \neq-1$

$\neg(0<-1)$

$1 \neq-1$

$\infty \neq-1$

$0 \neq-\infty$

$-0=0$

$-\infty<0$ 
$\neg(0<-\infty)$

$1 \neq-\infty$

$\infty \neq-\infty$

$\Phi \neq-\infty$

\subsubsection{General ordering}

$\neg(a<a)$

$(\operatorname{sgn}(a)=-1) \equiv(a<0)$

$(\operatorname{sgn}(a)=1) \equiv(0<a)$

$(\operatorname{sgn}(a)=0) \equiv(a=0)$

$(\operatorname{sgn}(a)=\Phi) \equiv(a=\Phi)$

$\neg(\Phi<a)$

$\neg(a<\Phi)$

$(a<\infty) \equiv((a \neq \infty) \wedge(a \neq \Phi))$

$(-\infty<a) \equiv((a \neq-\infty) \wedge(a \neq \Phi))$

\subsubsection{General algebraic properties}

$$
\begin{aligned}
& (a+b=\Phi) \equiv(a=\Phi) \vee(b=\Phi) \vee((a=\infty) \wedge(b=-\infty)) \vee((a=-\infty) \wedge(b=\infty)) \\
& (a-b=\Phi) \equiv(a=\Phi) \vee(b=\Phi) \vee((a=\infty) \wedge(b=\infty)) \vee((a=-\infty) \wedge(b=-\infty)) \\
& a+\infty=(\text { if }(a=\Phi) \vee(a=-\infty) \text { then } \Phi \text { else } \infty) \\
& a-\infty=(\text { if }(a=\Phi) \vee(a=\infty) \text { then } \Phi \text { else }-\infty) \\
& (a-b=0) \Rightarrow a=b \\
& (a+b=a+c) \Rightarrow(b=c):((a \neq \infty) \wedge(a \neq-\infty) \wedge(a \neq \Phi)) \\
& (b+a=c+a) \Rightarrow(b=c):((a \neq \infty) \wedge(a \neq-\infty) \wedge(a \neq \Phi)) \\
& (a+b=\infty) \equiv((a=\infty) \wedge(b \neq \Phi) \wedge(b \neq-\infty)) \vee((b=\infty) \wedge(a \neq \Phi) \wedge(a \neq-\infty)) \\
& (a+b=-\infty) \equiv((a=-\infty) \wedge(b \neq \Phi) \wedge(b \neq \infty)) \vee((b=-\infty) \wedge(a \neq \Phi) \wedge(a \neq \infty)) \\
& (a+b) \in R:(a \in R) \wedge(b \in R) \\
& -(a+b)=-a-b
\end{aligned}
$$


$(a-b<0) \equiv(a<b)$

$(a<b) \vee(a=b) \vee(b<a):(a \neq \Phi) \wedge(b \neq \Phi)$

$(0<a+b):(0<a) \wedge(0<b)$

$(0<a \times b):(0<a) \wedge(0<b)$

$(a \times b<0):(0<a) \wedge(b<0)$

$(a \times b<0):(a<0) \wedge(0<b)$

$\infty^{-1}=0$

$\left(a^{-1}=\Phi\right) \equiv(a=\Phi)$

$a^{-1} \neq 0:(a \neq \infty) \wedge(a \neq-\infty)$

$0<a^{-1}:(0<a) \wedge(a \neq \infty)$

\subsection{Hand Derivations}

The elementary proofs given here illustrate how standard proofs may be extended to the transreals. This section is intended as an introduction to transreal proof. No attempt is made to survey proofs or proof methods. As an exercise, the reader may care to check the mechanically generated theorems above.

As usual, hand proofs may assume associativity and commutativity, and may assume that the side conditions of a theorem are met when it is applied, but sometimes this is made explicit.

\subsubsection{Substitution}

Formal proofs are chains of substitutions grounded in axioms. Some theorems may be established by a chain of substitutions which does not involve any guarding clauses. The following proof is a very simple example of this. This proof was checked by machine at [T69]. Hence theorems [T69] and [T73] are identical.

Theorem: $\infty^{-1}=0$.

Proof: $\infty^{-1}=\left(0^{-1}\right)^{-1}[\mathrm{~A} 20]=0[\mathrm{~A} 19]$.

\subsubsection{Explicit Recognition of a Guarding Clause}

Some theorems require that a guarding clause is acknowledged explicitly, but are then obtained by a single chain of substitutions.

Theorem: $-0=0$.

Proof: $0-0=0[\mathrm{~A} 8]$ and the conditions on [A8] do not apply because $0 \neq \pm \infty, \Phi$ by the distinctness result obtained by machine derivation. But then $0+(-0)=0$ [A6] whence $-0=0$ [A3]. 


\subsubsection{Standard Theorem Holds for all Transreal Numbers}

Sometimes a standard theorem of real arithmetic holds for all transreal numbers without change. In the following proof the additive inverse is unique wherever it exists for transreal numbers. Of course, the additive inverse is closed in real arithmetic, but open in transreal arithmetic. However, the multiplicative inverse is open in both arithmetics and has more open cases in transreal arithmetic than in real arithmetic. Both standard theorems of uniqueness have a guarding clause "the inverse exists" and, as it happens, this guarding clause is sufficient in the transreal cases. Hence, the theorem is stated identically in the real and transreal cases, but the transreal cases have more content than the real ones. In the physical sciences it is commonly the case that a new theory has more content than an old one, even where the same terms are involved in the theory. Mathematics, too, is replete with such examples.

Theorem: If it exists, the additive inverse is unique.

Proof: We say that $b$ is an additive inverse of $a$ iff $a+b=0=b+a$. Suppose that $a+c=0=c+a$. Then $b=0+b$ [A3] $=c+a+b$ [by hypothesis] $=c+0$ [by definition] $=0+c$ [A2] $=c$ [A3]. Hence $b=c$ is the unique additive inverse wherever it exists.

Theorem: If it exists, the multiplicative inverse is unique.

Proof: We say that $b$ is a multiplicative inverse of $a$ iff $a \times b=1=b \times a$. Suppose that $a \times c=1=c \times a$. Then $b=1 \times b$ [A14] $=c \times a \times b$ [by hypothesis] $=c \times 1$ [by definition] $=1 \times c$ [A13] $=c$ [A14]. Hence $b=c$ is the unique multiplicative inverse wherever it exists.

\subsubsection{A Standard Theorem Holds for all Reals but has a Distinct Result for Strictly Transreal Parameters}

Sometimes a standard theorem holds for all real parameters, but has a distinct result for strictly transreal parameters. Both branches of the theorem need to be established. We begin with the standard theorem.

Theorem: $0 \times a=a \times 0=0: a \neq \pm \infty, \Phi$.

Proof: Firstly $0 \times a=a \times 0$ [A13]. Secondly, hypothesise that there is some real $(a \times 0)$. Then $0=(a \times 0)-(a \times 0)$ [A8] $=(a \times(0+0))-(a \times 0)$ [A3] $=(a \times 0)+(a \times 0)-(a \times 0)$ [A31] $=(a \times 0)+0$ [A8] $=a \times 0$ [A2] [A3]. This establishes the equality $0=a \times 0$ subject to the hypothesis that $a \times 0$ is real. But 0 is real, hence $(a \times 0)$ is real by the equality $0=a \times 0$, and this discharges the hypothesis. This completes the proof.

We now deal with the non-standard theorem by surveying cases.

Theorem: $0 \times a=a \times 0=\Phi: a= \pm \infty, \Phi$.

Proof: Firstly $0 \times a=a \times 0$ [A13]. Secondly, suppose $a=\Phi$ then $\Phi \times 0=\Phi$ [A15], as required. Thirdly, suppose $a=\infty$ then $\infty \times 0=\Phi$ [A16], as required. Fourthly, suppose $a=-\infty$ then $-\infty \times 0=(-1 \times \infty) \times 0[\mathrm{~T} 30]=-1 \times \Phi[\mathrm{A} 16]$ $=\Phi \times(-1)[\mathrm{A} 13]=\Phi[\mathrm{A} 15]$, as required. This completes the proof.

\subsubsection{The Method of Exclusion}

Transreal arithmetic is total so every well formed formula involving only literal numbers, the arithmetical operators, and brackets, can be evaluated. Given that a result exists, it is sometimes possible to categorise it by excluding cases. This is particularly easy where the cases are set out by quadrachotomy [A30].

Theorem: $a^{-1}<0:(a<0) \wedge(a \neq-\infty)$

Proof: The parameter $a$ is given, hence $a^{-1}$ exists by totality. By quadrachotomy exactly one of $a^{-1}<0, a^{-1}=0$, $a^{-1}>0, a^{-1}=\Phi$.

We begin by excluding the guarding clause. Firstly, suppose $a=-\infty$ then $a^{-1}=(-\infty)^{-1}=0[\mathrm{~A} 21]$ and $\neg(0<0)[\mathrm{T} 18]$ so this case is excluded. 
We then exclude all but one of the cases in the quadrachotomy. Secondly, suppose $a=0$ then $a^{-1}=0^{-1}=\infty$ [A20] but $\neg(\infty<0)$ [A25] so this case is excluded. Thirdly, suppose $a>0$ then $a^{-1}>0$ [T72] hence $\neg\left(a^{-1}<0\right)$ [A30] so this case is excluded. Fourthly, suppose $a=\Phi$ then $a^{-1}=\Phi^{-1}=\Phi[$ A21] but $\neg(\Phi<0)$ [T21] so this case is excluded. This leaves only $a^{-1}<0$ which completes the proof.

\subsubsection{A Standard Theorem Requires a Non-Standard Guarding Clause}

Sometimes a standard theorem requires a non-standard guarding clause, which may be found by enumerating all combinations of real and strictly transreal variables. The proof method is to establish the theorem on real numbers, and then to test all of the remaining, strictly trans-real cases. As an exercise, the reader may care to check the guarding clause on the distributivity axiom [A31] by assuming distributivity over purely real parameters and then examining all combinations of parameters involving strictly transreal numbers. There are many symmetries in these cases which makes the proof much less cumbrous than might, at first, appear.

Now, however, we establish a standard theorem involving reciprocals of real numbers. The guarding clause excludes all strictly transreal numbers and the number zero.

Theorem: $(a \times b)^{-1}=b^{-1} \times a^{-1}: a, b \neq 0, \pm \infty, \Phi$.

Proof: $(a \times b)^{-1}$ is a multiplicative inverse of $a \times b$. And $(a \times b) \times\left(b^{-1} \times a^{-1}\right)=a \times\left(b \times b^{-1}\right) \times a^{-1}=a \times 1 \times a^{-1}$ because $b$ and $b^{-1}$ are multiplicative inverses. Similarly, $a \times 1 \times a^{-1}=a \times a^{-1}=1$ because $a$ and $a^{-1}$ are multiplicative inverses. Hence, $b^{-1} \times a^{-1}$ is a multiplicative inverse of $a \times b$. But the multiplicative inverse is unique [T76] so $(a \times b)^{-1}=b^{-1} \times a^{-1}$ as required.

Having established the real theorem we then go on to examine all combinations of real and strictly transreal parameters. During this survey we may choose to take a greater or lesser advantage of symmetry, but on completing the proof we find that the new guarding clause [T81] is less restrictive than the guarding clause for the real case [T80]. This shows that we have derived a theorem over the transreals that is a strict extension of the equivalent law ranging over real numbers. Note that the transreal theorem deals with reciprocals, not just with multiplicative inverses.

Theorem: $(a \times b)^{-1}=b^{-1} \times a^{-1}: \neg(a=0 \wedge-\infty<b<0), \neg(b=0 \wedge-\infty<a<0)$.

Proof: Firstly, [T80] establishes the theorem for real $a, b \neq 0$. It remains to establish the theorem for all combinations of transreal parameters with $a$ or $b$ strictly transreal or zero.

Secondly, suppose $a=\Phi$ then LHS $=(\Phi \times b)^{-1}=\Phi^{-1}$ [A15] $=\Phi\left[\right.$ A22] and RHS $=b^{-1} \times \Phi^{-1}=b^{-1} \times \Phi[$ A22] $=\Phi$ [A13] [A15]. Hence, LHS = RHS as required. Similarly for all combinations of parameters with $a=\Phi$ or $b=\Phi$. Note that the theorem holds in all of these cases where nullity behaves like the object bottom in some algebras. ${ }^{9,6}$ But also note that nullity is not identical to bottom so this behaviour cannot be assumed and must be demonstrated, as just done.

Having disposed of all combinations of real and strictly transreal parameters involving the strictly transreal number nullity, we must now deal with all combinations of parameters involving the infinities.

We begin by considering the cases where both parameters are an infinity. Thirdly, suppose $a=b=\infty$ then LHS $=(\infty \times \infty)^{-1}=\infty^{-1}$ [A23] $=0$ [A20] and RHS $=\infty^{-1} \times \infty^{-1}=0 \times 0$ [A20] $=0$ [T77]. Hence, LHS $=$ RHS as required. Similarly for all combinations of $a= \pm \infty$ when $b= \pm \infty$.

We then consider the cases where one parameter is zero and the other is an infinity. Fourthly, suppose $a=0, b=\infty$ then LHS $=(0 \times \infty)^{-1}=\Phi^{-1}$ [A13] [A16] $=\Phi\left[\right.$ [A22] and RHS $=\infty^{-1} \times 0^{-1}=0 \times \infty$ [T73] [A20] $=\Phi$ [A13] [A16]. Hence, LHS $=$ RHS as required. Similarly for all cases where exactly one of $a, b=0$ when exactly one of $a, b= \pm \infty$. 
We then consider the cases where one parameter is zero and the other is real and positive. Fifthly, suppose $a=0,0<b<\infty$ then LHS $=(0 \times b)^{-1}=0^{-1}[\mathrm{~T} 77]=\infty$ [A20] and RHS $=b^{-1} \times 0^{-1}=b^{-1} \times \infty$ [A20] $=\infty$ [T72] [A13] [A23]. Hence, LHS $=$ RHS as required. Similarly for all cases where exactly one of $a, b=0$ when exactly one of $0<a, b<\infty$.

We then consider the cases where one parameter is zero and the other is real and negative. Sixthly, suppose $a=0,-\infty<b<0$ then LHS $=(0 \times b)^{-1}=0^{-1}\left[\right.$ T77] $=\infty$ [A20] and RHS $=b^{-1} \times 0^{-1}=b^{-1} \times \infty$ [A20] $=-\infty$ [T79] [A13] [A23]. Thus LHS $\neq$ RHS, which is consistent with the guarding clause. Similarly for all cases where exactly one of $a, b=0$ when exactly one of $-\infty<a, b<0$.

Finally, we consider the case where both parameters are zero. Seventhly, suppose $a=0, b=0$ then LHS $=(0 \times 0)^{-1}=0^{-1}$ [T77] $=\infty$ [A20] and RHS $=0^{-1} \times 0^{-1}=\infty \times \infty$ [A20] $=\infty$ [A25] [A23]. Hence, LHS $=$ RHS as required.

This completes the survey of cases and thereby completes the proof.

\section{Discussion}

As is well known, real arithmetic has a long and complicated history. In ancient Egypt, fractions of the form $1 / d$ with $d=1,2,3, \ldots$ were known, along with two special fractions $2 / 3$ and $3 / 4$. In ancient China, negative numbers were known, but zero was not. In ancient India, zero was known. At each stage in the development of mathematics it is tempting to assume that one's own mathematics has achieved the pinnacle of success, but this is rarely, if ever, the case. For example, contemporary real analysis recognises two special limits $\infty=1 / 0=k / 0$ when $k>0$ and $-\infty=-1 / 0=k / 0$ when $k<0.6$ These limits are not numbers, because they do not obey the axioms of real arithmetic, and because they have the peculiar property that they may be distinct, or not, as the case requires. ${ }^{6}$ More disturbingly, there is no number $\Phi=k / 0$ when $k=0 .{ }^{6}$ By contrast, transreal arithmetic defines the distinct numbers $\infty=1 / 0=k / 0$ when $k>0$ and $-\infty=-1 / 0=k / 0$ when $k<0$ and $\Phi=k / 0$ when $k=0$, thereby supplying the missing fractions. There appears to be a historical inevitability to this - the filling in of missing cases. Having obtained these missing fractions, the entire set of transreal numbers is used in transreal analysis ${ }^{3}$ where it extends and simplifies many important functions, such as the exponential, logarithm, and the trigonometric functions. In short, transreal numbers are a potentially useful extension of the real numbers.

Throughout its history, real arithmetic has developed as a codification of how people use arithmetic in their daily lives so it should not surprise us if human psychological limits have affected the structure of real arithmetic. Human limits are thrown into relief when we consider computer arithmetic. Real arithmetic is partial, it does not, for example, permit division by zero. In the past this was of no consequence to the majority of users of arithmetic. Excluding division by zero does make real arithmetic simple and fits well with the single stream of consciousness inherent in human psychology so that a human calculator can naturally devote the whole of his or her mental resources to the essence of a problem and does not risk overflowing his or her short-term memory by considering many branching borderline cases. By contrast, an electronic computer, whilst generally following a serial path of computation, finds it easy to consider a large number of trivial cases. It can easily process the additional algebraic rules for transarithmetic multiplication and division. While classical real arithmetic is the dominating arithmetical calculus, we argue that total systems like the transreals are worthy of consideration as an alternative model for computer arithmetic.

IEEE 754 floating-point arithmetic $^{8}$ is the most widely used form of computer arithmetic. Floating point units conforming to this standard are now part of almost all general-purpose computers, and programs using this arithmetic are put to work in many areas of daily life. All arithmetic operations in the standard are total in the sense that they yield a result. Normal floating point numbers are distinguished from special NaN ("NotANumber") elements which are returned by "invalid operations" such as division by zero or taking the square root of a negative number. NaNs come in a "quiet NaN" and a "signalling NaN" variety. There are two zeros ( +0 and -0$)$, and certain bit patterns are interpreted as positive and negative infinity. A defining characteristic is that an $\mathrm{NaN}$ value is not equal to any other value including itself, i.e. $\mathrm{NaN} \neq \mathrm{NaN}$.

The transreals do not deal with floating point numbers and as such can not be compared directly to the IEEE 754 standard. However, we argue that its treatment of classically invalid operations is simpler compared to that standard. In the 
transreals, a single new element (nullity) is added, unlike the different varieties of NaNs. Although nullity has special algebraic properties, i.e. it is propagated by the various arithmetic operations, it is a normal element in the sense that it is equal to itself. Equality is thus a reflexive operation, as usual in mathematics. We believe that this is a more intuitive approach which makes it easier for humans to reason about arithmetic operations.

Nullity is a special element of the transreals in the sense that it is different from all real numbers. This can be used to model "undefined values" in a way that "null" values are often used in programming. A database record with value nullity means that it has not been set to any real value. A time stamp with value nullity means that it has not been set to any real time, etc.

It should be stressed that the transreals are by no means the only total extension of the real numbers. A well known alternative approach is to add just a single new element. This corresponds to collapsing +infinity, -infinity and nullity into one element in the transreals. There are also various options for the topological structure of such real number extensions. The choices made in the design of the transreal number system were governed by our wish to model computer arithmetic and in particular to support the Perspex Machine.

We recognize that the presentation given here is limited. We have set out the axioms of transreal arithmetic and explained how they were checked by machine, but the details of that checking are available only in proof files on the world wide web. ${ }^{12}$ Similarly, giving machine generated theorems with no indication of the proof path is not helpful to the general reader. However, it is hoped that the hand proofs are assessable to all, and show how easy and effective it is to develop proofs in transreal arithmetic.

Because the variables in a transreal theorem can range over more elements compared to a corresponding real arithmetic rule, our theorems and axioms often have additional side conditions that exclude particular combinations of variable values for which the rule does not hold. Typical examples are the Additive Inverse [A8] or Distributivity [A31] axioms which have no side-conditions in the corresponding real arithmetic laws. On the other hand, any theorem with a variable that ranges over the full transreals expresses both a classical real arithmetic rule as well as infinity- and nullitypropagating properties. Combining these aspects into a single rule can help to streamline reasoning compared to a partial operation calculus where one first performs a calculation under the assumption that all expressions are defined, and then reasons separately about the cases where some expressions might have undefined values. On the other hand, a drawback of the additional expressive power in the transreals is that proofs can become longer due to the need to consider additional infinity and nullity cases for variables. These kind of tradeoffs are typical when a calculus with partial operations is extended to a total operation system.

\section{Conclusion}

We have presented an axiomatisation of the algebraic structure of transreal arithmetic. This is a calculus which extends the real numbers by a nullity element as well as $\infty$ and $-\infty$ so as to better reflect computer arithmetic. Transreal equality is a reflexive operation and there is only one element that does not belong to the extended real number line. These properties help to keep the reasoning about transreals simple and intuitive. The fact that all arithmetic operations are total is useful for the modelling of computer arithmetic where all operations have a result. Our experience with the transreals suggests the use of versions of floating-point arithmetic that do not have arithmetical exceptions and in which every number is a first-class citizen.

Compared to classical real arithmetic, transreal arithmetic boasts theorems that are more expressive than their traditional equivalents in that they combine real number laws with infinity- and nullity-propagating properties of arithmetic operations. The added expressivity comes at the price of a more complicated number system where proofs have to consider additional cases. It will be interesting to see whether total systems of arithmetic become more popular in the future, given the totality of operations in some computer arithmetic systems and the need to consider undefined values when reasoning about computer programs. 


\section{Acknowledgement}

We would like to thank Stefan Karpinski, PhD Candidate, Department of Computer Science, University of California, Santa Barbara, for pointing out a typographical error in theorem [T53] as it appeared in a pre-publication version of this paper.

\section{References}

1 J.A.D.W. Anderson, "Representing Geometrical Knowledge" Phil. Trans. Roy. Soc. Lond. series B, vol. 352, no. 1358, pp. 1129-1139, Aug. (1997).

2 J.A.D.W. Anderson, "Perspex Machine VII: The Universal Perspex Machine" in Vision Geometry XIV Longin Jan Latecki, David M. Mount, Angela Y. Wu, Editors, Proceedings of the SPIE Vol. 6066 (2006).

3 J.A.D.W. Anderson, "Perspex Machine IX: Transreal Analysis" in Vision Geometry XV Longin Jan Latecki, David M. Mount, Angela Y. Wu, Editors, Proceedings of the SPIE, this volume (2007).

4 S. Noble, B.A. Thomas, J.A.D.W. Anderson, "Perspex Machine X: Software Development" in Vision Geometry XV Longin Jan Latecki, David M. Mount, Angela Y. Wu, Editors, Proceedings of the SPIE, this volume (2007).

5 F. Ayres, Modern Abstract Algebra, McGraw-Hill (1965).

6 M. Beeson, F. Wiedijk, "The Meaning of Infinity in Calculus and Computer Algebra Systems" in J. Symbolic Computation vol. 39, pp. 523-538 (2005).

7 J.H. Conway, On Numbers and Games, 2nd Edition, A.K. Peters Ltd (2001).

8 IEC 60559:1989, Binary floating-point arithmetic for microprocessor systems.

9 S.C. Kleene, Introduction to Mathematics, van Nostrand 1950.

10 Paulson, L "Isabelle: the next seven hundred theorem provers" in CADE9, pp. 772-773 (1988).

11 T. Nipkow, L.C. Paulson, M. Wenzel, Isabelle/HOL - A Proof Assistant for Higher-Order Logic, Springer (2002).

12 At the time of the conference, The Isabelle/HOL proof files will be available at: http://www.bookofparagon.com/Pages/Downloads.htm 